\title{
Comparison of IOL Power Calculated by Preoperative Biometry versus Intraoperative Wavefront Aberrometry in Thai Cataract Patients.
}

Bharkbhum Khambhiphant ( $\nabla$ bharkbhum@gmail.com )

Chulalongkorn University https://orcid.org/0000-0003-3167-9186

Sribenjapanon Thanyaporn

Chulalongkorn University

Research article

Keywords: Wavefront aberrometry, biometry, IOL power calculation

Posted Date: August 21st, 2020

DOI: https://doi.org/10.21203/rs.3.rs-25811/v4

License: (c) (1) This work is licensed under a Creative Commons Attribution 4.0 International License.

Read Full License 
1 Comparison of IOL Power Calculated by Preoperative Biometry versus

2 Intraoperative Wavefront Aberrometry in Thai Cataract Patients.

3 Khambhiphant Bharkbhum, MD

4 Department of Ophthalmology, King Chulalongkorn Memorial Hospital, Thai red

5 Cross Society, Bangkok, Thailand.

6 Department of Ophthalmology, Faculty of Medicine, Chulalongkorn University,

7 Bangkok, Thailand.

$8 \quad$ Email: bharkbhum@gmail.com

9 Sribenjapanon Thanyaporn, MD

10 Department of Ophthalmology, Faculty of Medicine, Chulalongkorn University,

11 Bangkok,

12 Thailand.

13 Email: boedcea@gmail.com

14 Total Abstract word count: 245

15 Total Maintext word count: 2,637

16 Correspondence: Bharkbhum Khambhiphant, MD, Department of Ophthalmology,

17 King Chulalongkorn Memorial Hospital, Thai red Cross Society, 1873 Rama 4

18 Road, Pathumwan, Bangkok 10330. Thailand.

19 Tel. +66898111573 Fax.+6622528290 Email: bharkbhum@gmail.com 
1 Comparison of IOL Power Calculated by Preoperative Biometry versus

2 Intraoperative Wavefront Aberrometry in Thai Cataract Patients.

3 Khambhiphant Bharkbhum, MD , Sribenjapanon Thanyaporn, MD

5 Abstract

6 Background: As people now are expecting more precise and predictable results

7 from cataract surgery. We need to find agreement between the calculated

8 intraocular lens (IOL) power from using the SRK/T based preoperative biometry

9 and the intraoperative wavefront aberrometry $\left(O R A^{\circledR}\right)$ in Thai cataract patients.

10 Methods: Eyes that underwent cataract surgery with monofocal or multifocal IOL

11 implantation were enrolled in this prospective study. All eye biometry was

12 measured preoperatively and the ORA intraoperatively. The SRK/T suggested IOL

13 from the preoperative biometry was chosen in all cases. The suggested power and

14 the estimated refraction (EST) from both devices were collected. Bland Altman

15 analysis was used to find the agreement between them. The predicted EST of

16 implanted IOL from both devices were compared with the one-month

17 postoperative SE.

18 Results: The study comprised 97 eyes (79 patients). Of these, 38 eyes $(39.2 \%)$

19 had the same suggested IOL power, 36 eyes (37.1\%) were within $\pm 0.5 \mathrm{D}, 20$ eyes

$20(20.6 \%)$ were within $\pm 1.0 \mathrm{D}$ and 3 eyes were beyond $\pm 1.0 \mathrm{D}$. Bland-Altman

21 analysis found the mean difference between IOL power calculated from both

22 devices was 0.39 with LoA of -0.54 to 1.31 . The correlation was $98.50 \%(95 \% \mathrm{Cl}$

$2398 \%-99.10 \%)$. In the same suggested IOL power group, the median difference of

24 EST by preoperative biometry and ORA compared with one-month postoperative

25 SE were $-0.08(95 \% \mathrm{Cl}:-0.08,1.11)$, and $-0.14(95 \% \mathrm{Cl}:-0.88,1.2)$, respectively. 
1 Conclusions:The ORA and preoperative biometry results were in concordance

2 with each other. The result of preoperative biometry was more accurate than ORA

3 in this study.

4 Trial Registration: The thai clinical trial registration

5 number: TCTR20171005001

6 Registration Date: October $3^{\text {rd }}$, 2017

7 First Enrollment: November 10 $10^{\text {th }}, 2017$

8 Keywords: Wavefront aberrometry, biometry, IOL power calculation

9

10

11

12

13

14

15

16

17

18

19

20

21

22

23

24

25 


\section{Background}

Cataract is the leading cause of reversible blindness worldwide. The World

3 Health Organization(WHO) has estimated that in 2020, 32 million cataract

4 operations will be performed. ${ }^{1}$ People now are expecting more precise and

5 predictable results from cataract surgery. With advanced technologies and

6 surgical techniques, outcome of cataract surgery was expected as a refractive

7 surgery. ${ }^{2}$ Therefore, routine preoperative biometry (Axial length, keratometry, etc.)

8 plays an important role in enabling the accuracy of IOL power calculation which

9 essential in good refractive outcome. ${ }^{3}$

$10 \quad$ Ultrasound biometry (A-scan) has been used to measure the ocular axial

11 length $(A L)$ which requires a technician's skill. ${ }^{3}$ We found that the $A L$

12 measurement was shorter in contact or applanation ultrasound compared with

13 non-contact or immersion ultrasound in prior studies. ${ }^{2,4,5}$ Since 2000 , optical

14 biometry has become the new standard for measuring the axial length and other

15 ocular parameters. The latest generation IOLmaster ${ }^{\circledR} 700$ (Carl Zeiss Meditec AG,

16 Germany), which is a Swept source OCT with B-scan biometry-based device ${ }^{6}$, is

17 now one of the standards for pre-operative biometry. Various studies show that

18 the IOLmaster ${ }^{\circledR 700}$ results are more precise and repeatable than ultrasound

19 biometry. ${ }^{7-10}$

20 Even though the IOL power can be calculated in eyes with abnormal axial

21 length, prior to keratorefractive surgery and toric intraocular lens implantation

22 there are still challenges as they are prone to have prediction error. ${ }^{11}$ Therefore,

23 another method is needed which can give us the precise ocular biometry

24 especially intraoperatively after lens removal. The optiwave refraction analysis

25 (ORA system ${ }^{\circledR}$ with VerifEye ${ }^{\mathrm{TM}}$ Alcon Laboratories, Inc., Tx, USA) is the latest 
1 technology using wave-front aberrometry refraction intraoperatively in phakic,

2 aphakic or pseudophakic stages which promise to be more accurate for IOL

3 selection and positioning compared to standard methods. ${ }^{12-14}$ It gives the

4 intraoperative $\mathrm{IOL}$ calculation power and the postoperative refraction at the end of

5 surgery. This seems to satisfy the expectations of the patients.

6 This study was designed to find the agreement between the calculated

7 intraocular lens (IOL) power by using the SRK/T based preoperative biometry (IOL

8 master ${ }^{\circledR 700)}$ ) and the intraoperative wave-front aberrometry (ORA system ${ }^{\circledR}$ with

9 VerifEye ${ }^{\mathrm{TM}} 2017$ Alcon Laboratories, Inc., Tx, USA) in Thai cataract patients. We

10 also compared the estimated postoperative refraction results from each device at

11 one month to determine their accuracy.

13 Methods

14 Patients

Ninety-seven eyes from 79 patients that underwent cataract surgery at the

16 King Chulalongkorn Memorial Hospital performed by a single surgeon (KB) with monofocal or multifocal IOL implantation were enrolled in this prospective nonrandomized study. The Institutional Review Board (IRB) of the Faculty of Medicine, Chulalongkorn University, has approved the study. Inclusion criteria were scheduled cataract patients over 18 years who

21 underwent cataract surgery by phacoemulsification and femtosecond laser assisted phacoemulsification with non-toric monofocal and multifocal IOL implantation within the bag. All cases were planned to have an estimated

24 refraction close to plano ( 0 diopter). Exclusion criteria were patients who have

25 limitation of using the IOL Master ${ }^{\circledR} 700$ (Densely opacities media/mature cataract), 
1 conditions that was not suitable for using intraoperative aberrometry (corneal scar,

2 small pupil size less than $5.0 \mathrm{~mm}$., macular and retinal abnormality, inability to fix

3 intraoperative aberrometry aiming beam, etc), previous refractive surgery history,

4 intraoperative complications (ruptured posterior capsule, dropped nucleus, etc).

\section{Surgery}

6 Once the written informed consent was obtained, each eye was completely

7 examined by slit lamp and auto kerato-refractometer (Auto Kerato-refractometer

8 KR-800; Topcon Co, Tokyo, Japan) under standard preoperative assessment.

9 During the first visit each preoperative biometry was measured with the IOL

10 Master $^{\circledR} 700$ before any ocular contacts such as mydriatric and other topical drugs

11 instillation. In this study the SRK/T suggested IOL power and the estimated postoperative refraction that targeting emmetropia from the preoperative biometry was chosen in all cases and represented as the IOL master 700 calculated or

14 chosen power.

On the operating day each eye was routinely prepared with topical antibiotic (0.5\%Moxifloxacin eye drop 1 drop every 15 minutes for 1 hour preoperatively), mydriatric drug (1\% Tropicamide eye drop 1 drop every 15 minutes for 1 hour preoperatively). All surgeries were performed under topical anesthesia $(0.5 \%$ Tetracaine eye drop 1 drop every 10 minutes for half an hour preoperatively).

20 Each eye was also prepared with the same technique by draping with

21 Opsite $^{\mathrm{TM}}$ (Smith \& Nephew, Hull, UK) and using the Lieberman adjustable temporal speculum size ( E40-100 adult size; PMS, Germany) in order not to apply excessive pressure to the eye. Phacoemulsification was performed with temporal clear corneal incision ( $2.75 \mathrm{~mm}$ wound size) technique. The incision site was approximately aligned at $180^{\circ}$. The 2 side-port were opened by using $1 \mathrm{~mm}$. slit 
1 knife at 90 and 225 degree respectively. The circular capsulorrhexis size aiming

$25.5 \mathrm{~mm}$ was created by no.27 needle tip in all cases under Provisc ${ }^{\circledR}\left(\right.$ Provisc $^{\circledR}$;

3 Alcon, Tx, USA). The standard phacoemulsification with vertical chopped

4 technique was used in all cases.

5

6 treatment was performed with the LenSx laser (Alcon Laboratories, Fort Worth,

7 TX, USA). Each eye was prepped as Phacoemulsification group. After

8 femtosecond laser precut was completed, phacoemulsification was performed

9 using the Centurion Vision System (Alcon Laboratories). The surgery was then

10 completed with standard phacoemulsification procedure with vertical chopped

11 technique.

After the lens nucleus and cortex were removed, we inflated Provisc ${ }^{\circledR}$ to maintain the ocular volume. During the ORA measurement, the Provisc ${ }^{\circledR}$ was used as the ophthalmic viscoelastic device (OVD) of choice for maintaining normotensive level of eye pressure during the measurement in all cases. The IOP checked with a Barraquer tonometer was not to exceed $20 \mathrm{mmHg}$ before start measured with the ORA. The ORA was measured once in the aphakic stage to derive the suggested IOL power and estimated postoperative refraction in all cases and represented as the ORA suggested IOL power. All eyes were implanted with preoperative chosen foldable monofocal IOL and multifocal IOL power suggested by IOL Master ${ }^{\circledR} 700$ in the bag due to the ethical considerations. Wounds ware closed with corneal stromal hydration. All patients had standard routine postoperative follow up.

The suggested IOL power and the estimated refraction (EST) from both devices were collected. We also collected the postoperative auto refraction (Auto 
1 Kerato-Refractometer KR-800; Topcon Co, Tokyo, Japan) results at approximately

2 1day, and 1 month for determining the accuracy of both devices. The UCVA and

3 BCVA of the eyes at each visit were collected.

4 Statistical analysis

5 Bland Altman analysis was used to find the agreement between them.

6 Subgroup analysis in eyes with the same IOL power reading from both devices

7 were analyzed. The predicted estimated refraction of implanted IOL power from

8 both devices were compared with the one-month postoperative Spherical

9 equivalent from auto-keratorefractometer.

10 Results

11 This study comprised 79 subjects with 97 eyes of which 65 eyes received a

12 monofocal IOL ( ALCONSA60WF, HOYAIMICS250, HOYAIMICS251 or

13 ACRYSOFMA60AC) and 32 eyes received a multifocal diffractive IOL (

14 ATLISATRI839 ). All eyes met the inclusion criteria and completed the follow-up

15 as set in the protocol. The characteristics of population are shown in Table1.

16 
1 Table 1 Demographics of the study population

2

\begin{tabular}{|c|c|c|}
\hline Characteristics & $\mathbf{N}$ & $\%$ \\
\hline \multicolumn{3}{|l|}{ Gender } \\
\hline Male & 21 & 26.6 \\
\hline Female & 58 & 73.4 \\
\hline Total & 79 & \\
\hline Age (year) & $67.3 \pm 6$ & \\
\hline \multicolumn{3}{|l|}{ Eye } \\
\hline OD & 45 & 46.4 \\
\hline OS & 52 & 53.6 \\
\hline Total & 97 & \\
\hline Axial length (mm) & $23.60 \pm 1.23$ & \\
\hline \multicolumn{3}{|l|}{ IOL } \\
\hline Monofocal & 65 & 67.0 \\
\hline Multifocal & 32 & 33.0 \\
\hline \multicolumn{3}{|l|}{ Type of Surgery } \\
\hline Phacoemulsification & 87 & 89.7 \\
\hline $\begin{array}{l}\text { Femtosecondlaser-assisted } \\
\text { phacoemulsification }\end{array}$ & 10 & 10.3 \\
\hline Total & 97 & \\
\hline
\end{tabular}


Of these, the A constant of each varied as 118.7 for ALCONSA60WF,

2118.4 for HOYAIMICS250/251and ACRYSOFMA60AC for monofocal IOL group.

3 The A constant of ATLISATRI839MP was 118.3.

We found that 38 eyes (39.2\%) had the same suggested IOL power from

5 the IOL master and the ORA whereas 36 eyes $(37.1 \%)$ were within $\pm 0.5 \mathrm{D}, 20$

6 eyes (20.6\%) were within $\pm 1.0 \mathrm{D}$ and 3 eyes were beyond $\pm 1.0 \mathrm{D}$, consecutively as

7 shown in Table 2.

8

\begin{tabular}{|r|c|c|}
\hline $\begin{array}{r}\text { Difference of IOL power from } \\
\text { both devices }\end{array}$ & Eyes(N=97) & \\
\hline Chosen IOL=Suggested IOL & 38 & 39.2 \\
power & & 76.3 \\
\hline within $\pm 0.5 \mathrm{D}$ & 74 & 96.9 \\
\hline within $\pm 1.0 \mathrm{D}$ & 94 & 3.1 \\
\hline$> \pm 1.0 \mathrm{D}$ & 3 & \\
\hline
\end{tabular}

9

10 Table 2. Differences of IOL power between preoperative biometry ( IOL Master ${ }^{\circledR}$ )

11 or chosen power and Intraoperative aberrometry (ORA ${ }^{\circledR}$ )

The IOL powers calculated from IOL master and the ORA were used in the analysis. The Bland-Altman analysis found the mean difference between IOL

14 power calculated from both devices was 0.39 with LoA of -0.54 to 1.31. The correlation was 98.50\% (95\%Cl 98\%-99.10\%) as shown in Fig 1. 


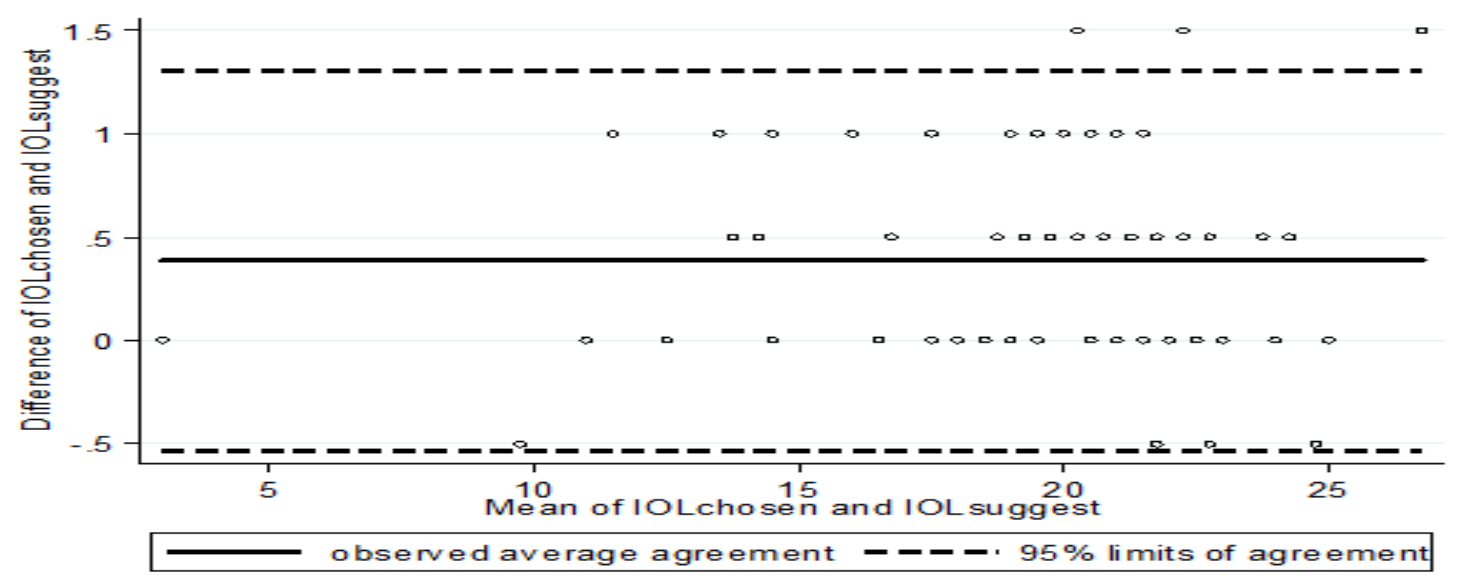

2 Figure 1 Bland-Altman analysis shows the difference between the IOL power

3 derived from both devices is plotted against the mean for the two devices. The

4 dotted lines represent the $95 \%$ limits of agreement.

Since we wanted to know the kind of calculation that gives us the closest to

6 refractive target (plano). Since this was the first project using the ORA in the

7 country and due to ethics considerations the IOL master calculated power was the

8 chosen as the IOL implanted in this study. We analysed only the same suggested

9 IOL power group and found that the median difference of estimated refraction by

10 preoperative optical biometry (IOL master $\left.{ }^{\circledR} 700\right)$ and intraoperative wavefront

11 aberrometry (ORA system ${ }^{\circledR}$ with VerifEye ${ }^{\mathrm{TM}}$ ) compared with one-month

12 postoperative spherical equivalent by auto refraction were $-0.08(95 \% \mathrm{Cl}:-0.08$,

$131.11)$, and $-0.14(95 \% \mathrm{Cl}:-0.88,1.2)$ respectively with statistical significance. A

14 total of 75 eyes (77\%) achieved a visual acuity of $20 / 20$.

\section{Discussion}

As the advance in cataract surgery technology is moving forward. We have

18 entered an era of precise and predictable outcome of the cataract surgery. ${ }^{15}$ Both 
1 preoperative biometry and IOL calculation are important factors for cataract

2 surgery. ${ }^{16}$ The new generation of formulas are being used for reaching the

3 refractive target, but they also have some limitations especially in the post

4 keratorefractive eyes and abnormal axial length. The non-contact optical biometry

5 has been routinely used worldwide due to its precision. Because not only the

6 monofocal IOL is the IOL of choice for the patients, but also multifocal and Toric

7 IOL that need to be considered. Intraoperative wave-front aberrometry (ORA

8 system ${ }^{\circledR}$ with VerifEye ${ }^{\mathrm{TM}}$ ) is a new technology that provides more accuracy and is

9 promising for IOL power calculation especially in toric IOL implantation and post-

10 refractive surgery patient. ${ }^{13-17}$ It might also help the cataract surgeons work with

11 confidence.

In our study the subjects were implanted with non-toric IOL, and most of

13 them had normal axial length eyes (within $22 \mathrm{~mm} .-25 \mathrm{~mm}$ ). ${ }^{18}$ There were 38 eyes

$14(39.2 \%)$ that had the same suggested IOL power whereas 74 eyes $(76.3 \%)$ were

15 within $\pm 0.5 \mathrm{D}$. From the Bland-Altman analysis the correlation between both

16 devices was $98.50 \%$. We also found that the ORA result is inconcordance with

$17 \mathrm{IOL}$ master ${ }^{\circledR} 700$ which is the current gold standard for modern optical biometry

18 devices. ${ }^{19}$ Comparing with Zhang et al, they found that $46.9 \%$ (107 from 228 eyes)

19 had same recommended IOL power from ORA and IOL master 300 or IOL master

$20500 .{ }^{14}$ And the ORA postoperative refractive outcomes were comparable to

21 conventional biometry for monofocal IOL selection. ${ }^{14}$ Another previous study was

22 from Davison J. A. et al, a retrospective review in uncomplicated cataract surgery

23 with no previous ocular surgery found that $46 \%$ had same recommend IOL power

24 from IOLmaster700 and ORA, though this percentage was lower in the multifocal

$25 \mathrm{IOL}$ group. ${ }^{20}$ Fisher et al ${ }^{21}$, reported $39 \%$ of 44 post-lasik eyes that had the same 
1 suggested IOL power from the IOL master and the ORA. The limited number of

2 subjects could have made our results lower than the others. And we also included

3 the monofocal and multifocal IOL in the same group.

In 38 eyes which both the ORA and IOL master calculated the same IOL

5 power were analysed. The estimated refraction (EST) of those were different in

6 the same reading group. We found that the median differences of EST by

7 preoperative biometry and ORA compared with one-month postoperative SE were

$8-0.08(95 \% \mathrm{Cl}:-0.08,1.11)$, and $-0.14(95 \% \mathrm{Cl}:-0.88,1.2)$ respectively. The IOL

9 master calculated power (the chosen power) gave the closer estimate refraction

10 compare to autorefraction at 1 month post-operative than the ORA. Cionni et al

11 found that the ORA mean absolute prediction error was lower than the

12 preoperative calculation, $0.30 \mathrm{D} \pm 0.26(\mathrm{SD})$ versus $0.36 \pm 0.32 \mathrm{D}(p<0.0001)$.

13 And the absolute median prediction error was lower than the preoperative

14 calculation, $0.24 \mathrm{D}$ versus $0.29 \mathrm{D}(p<0.0001) .^{22}$

15 In our study, however, there were $77 \%$ of the subjects who achieved

16 uncorrected distance visual acuity of $20 / 20$. Although it was statistically significant

17 with the median difference of were -0.08 and -0.14 respectively. But it may not be

18 clinical significant due to the availability of IOL power in the market increments

19 step is $0.5 \mathrm{D}$.

20 In our study, we had a limited the number of subjects especially the post-

21 keratorefractive and Toric cases were not recruited. So we need further studies to

22 determine the accuracy of both devices in these groups. We also had the

23 heterogeneity of the study population i.e. more than one eye from each patient

24 was used, so some of the data is paired, the difference in IOL type (multifocal and 
14 monofocal IOL) and the difference kind of phacoemulsification ( Femtosecond

2 assisted and conventional phacoemulsification).

The factors that might affect the ORA results varied such as the eye lid speculum pressure that was applied to the eye during the ORA refraction, the ocular surface, type of viscoelastic, type of IOLs manufactured and surgeon experienced. Even though we used the Provisc OVDs and measured with the

7 Barraquae tonometer but we could only estimate that the pressure was around

$820 \mathrm{mmHg}$. We could not have a precise IOP at $20 \mathrm{mmHg}$ while measuring. The

9 clarity of the cornea and the pupil size were the factors that affected the IOL power

10 calculation by the ORA. We noticed that dry and cloudy cornea, the constricted

11 pupil less than $4.50 \mathrm{~mm}$ could not complete the measurement. The type of IOLs with different design, materials and A constant might also have had an effect on the calculated IOL power derived from the ORA while we use their EST refraction

14 to compare with the IOL master. We also found that the ORA could only be used with some IOL brands. So it needs numerous databases from multiple brands of IOL to be accumulated in their Analyzor ${ }^{\text {TM }}$ (Alcon Laboratories, Inc., Tx, USA) software. That means each surgeon can continuously optimizing IOL-specific lens constants that lead to progressively more accurate outcomes. In our study $10 \%$ of eyes were performed with FLACS eventhough only laser assisted capsulorhexis and lens fragmentation and it should be the other factor that affected. And to avoid the learning period factor, in our study the surgeon had experienced of using the ORA in more than 20 cases before the study started. The surgeon could do it with in $30 \mathrm{sec}$ average for the whole measurement.

The last conceptual problem is that final manifest refraction might have been measured at 3 months post-op, as after 1 month there might be still fluctuations in refraction. Nevertheless it is a good "real Life" and the information 
1 given is sufficient to give surgeons information how good the intraoperative

2 agreement between conventional and IA (ORA) calculation is. Also they can see

3 that absolute differences may be neglectable to a certain (clinical relevant) extent.

ORA itself can not only calculates the IOL power intraoperatively but it can also be used for refraction intraoperatively in supine position at the end of

6 surgery. It might help us find the estimated refraction at the end of the surgery.

7 However, this feature will need to be evaluated in terms of accuracy and precision.

\section{Conclusions}

The intraoperative aberrometry (ORA system ${ }^{\circledR}$ with VerifEye ${ }^{\mathrm{TM}}$ ) and preoperative optical biometry $\left(\mathrm{IOLmaster}{ }^{\circledR} 700\right)$ results were in concordance with each other. Although the result of preoperative biometry was more accurate than that of ORA in this study. ORA seems to be helpful in reassuring the IOL power

13 trend for the surgeon in difficult and complicated cases.

Abbreviations:

- Intraocular lens (IOL)

17 - Intraoperative wave-front aberrometry (ORA)

18 - Estimated Refractio (EST)

19 - Spherical equivalet (SE)

20 - Axial length (AL)

- Femtosecond laser assisted cataract surgery (FLACS)

- Ophthalmic viscosurgical device (OVD) 
1 Declarations

2 Ethics approval and consent to participate: IRB 694/59 from Faculty of

3 Medicine, Chulalongkorn University, Bangkok, Thailand.

4 The written informed consent was obtained from participants.

5

6 Consent for publication

$7 \quad$ Not applicable

$8 \quad$ Availability of data and material

9 The datasets generated and/or analysed during the current study are not publicly

10 available due to the government of Thailand policy but are only available from the

11 corresponding author on reasonable request.

12 Competing interests:

13 There are no financial disclosure in this study.

14 Funding: The Rachadapisek sompoch fund, Faculty of Medicine, Chulalongkorn

15 University, Bangkok, Thailand.

16 The funder just provided funding

17

18 Authors' contributions:

19 Coceptual design, Critical revision of Manuscript, Securing funding, Admin,

20 technical or material support, Supervision, Final Approval and Correspondig

21 author: BK ( Bharkbhum Khambhiphant, MD)

22 Data acquisition, Data Analysis/Interpretation, Drafting manuscript,

23 Statistical analysis : TS (Thanyaporn Sribenjapanon, MD)

24 All authors have read and approved the manuscript

25 Acknowledgement:

26 Not Applicable 


\section{$1 \quad$ References}

2 1. Agarwal A, Kumar DA. Cost-effectiveness of cataract surgery. Curr Opin

3 Ophthalmol. 2011;22(1):15-8.

4 2. Eleftheriadis H. IOLMaster biometry: refractive results of 100 consecutive

5 cases. Br J Ophthalmol. 2003;87(8):960-3.

6 3. Fontes BM, Fontes BM, Castro E. Intraocular lens power calculation by

7 measuring axial length with partial optical coherence and ultrasonic biometry. Arq

8 Bras Oftalmol. 2011;74(3):166-70.

9 4. Shammas HJ. A comparison of immersion and contact techniques for axial

10 length measurement. J Am Intraocul Implant Soc. 1984;10(4):444-7.

11 5. Giers U, Epple C. Comparison of A-scan device accuracy. J Cataract Refract

12 Surg. 1990;16(2):235-42.

13 6. Hirnschall N, Varsits R, Doeller B, Findl O. Enhanced Penetration for Axial

14 Length Measurement of Eyes with Dense Cataracts Using Swept Source Optical

15 Coherence Tomography: A Consecutive Observational Study. Ophthalmol Ther.

$162018 ; 7(1): 119-124$.

17 7. Jung S, Chin HS, Kim NR, Lee KW, Jung JW. Comparison of Repeatability and

18 Agreement between Swept-Source Optical Biometry and Dual-Scheimpflug

19 Topography.J Ophthalmol.2017;2017:1516395.

20 8. Yang JY, Kim HK, Kim SS. Axial length measurements: Comparison of a new

21 swept-source optical coherence tomography-based biometer and partial

22 coherence interferometry in myopia. J Cataract Refract Surg. 2017;43(3):328-32.

23 9. Calvo-Sanz JA, Portero-Benito A, Arias-Puente A. Efficiency and

24 measurements agreement between swept-source OCT and low-coherence 
1 interferometry biometry systems. Graefes Arch Clin Exp Ophthalmol.

2 2018;256(3):559-66.

3 10. Rose LT, Moshegov CN. Comparison of the Zeiss IOLMaster and applanation

4 A-scan ultrasound: biometry for intraocular lens calculation. Clin Exp Ophthalmol. $5 \quad 2003 ; 31(2): 121-4$.

6 11. Hemmati HD, Gologorsky D, Pineda R. Intraoperative wavefront aberrometry

7 in cataract surgery. Semin Ophthalmol. 2012;27(5-6):100-6.

8 12. Hatch KM, Woodcock EC, Talamo JH. Intraocular lens power selection and

9 positioning with and without intraoperative aberrometry. J Refract Surg.

$10 \quad 2015 ; 31(4): 237-42$.

11 13. lanchulev T, Hoffer KJ, Yoo SH, Chang DF, Breen M, Padrick T, et al.

12 Intraoperative refractive biometry for predicting intraocular lens power calculation

13 after prior myopic refractive surgery. Ophthalmology. 2014;121(1):56-60.

14 14. Zhang Z, Thomas LW, Leu SY, Carter S, Garg S. Refractive outcomes of

15 intraoperative wavefront aberrometry versus optical biometry alone for intraocular

16 lens power calculation. Indian J Ophthalmol. 2017;65(9):813-7.

17 15. Dick HB, Schultz T. A Review of Laser-Assisted Versus Traditional

18 Phacoemulsification Cataract Surgery. Ophthalmol Ther. 2017;6(1):7-18.

19 16. Hayek S, Kniestedt C, Barthelmes D, Sturmer J. Quality assurance in biometry

20 before cataract surgery: which patients have an increased risk of aberrance from

21 target refraction. Klin Monbl Augenheilkd. 2007;224(4):244-8.

22 17. Woodcock MG, Lehmann R, Cionni RJ, Breen M, Scott MC. Intraoperative

23 aberrometry versus standard preoperative biometry and a toric IOL calculator for

24 bilateral toric IOL implantation with a femtosecond laser: One-month results. J

25 Cataract Refract Surg. 2016;42(6):817-25. 
1 18. Hill DC, Sudhakar S, Hill CS, King TS, Scott IU, Ernst BB, et al. Intraoperative

2 aberrometry versus preoperative biometry for intraocular lens power selection in

3 axial myopia. J Cataract Refract Surg. 2017;43(4):505-10.

4 19. Akman A, Asena L, Gungor SG. Evaluation and comparison of the new swept

5 source OCT-based IOLMaster 700 with the IOLMaster 500. Br J Ophthalmol.

$6 \quad 2016 ; 100(9): 1201-5$.

7 20. Davison JA, Potvin R. Preoperative measurement vs intraoperative

8 aberrometry for the selection of intraocular lens sphere power in normal eyes. Clin

9 Ophthalmol. 2017;11:923-9.

10 21. Fisher B, Potvin R. Clinical outcomes with distance-dominant multifocal and

11 monofocal intraocular lenses in post-LASIK cataract surgery planned using

12 an intraoperative aberrometer. Clin Exp Ophthalmol. 2018;46(6):630-636.

13 22. Cionni RJ, Dimalanta R, Breen M, Hamilton C. A large retrospective database

14 analysis comparing outcomes of intraoperative aberrometry with conventional

15 preoperative planning.J Cataract Refract Surg. 2018;44(10):1230-1235. 
1 Figure 1 Bland-Altman analysis shows the difference between the IOL power

2 derived from both devices is plotted against the mean for the two devices. The 3 dotted lines represent the $95 \%$ limits of agreement.

4

5

6

7

8

9

10

11

12 
Figures

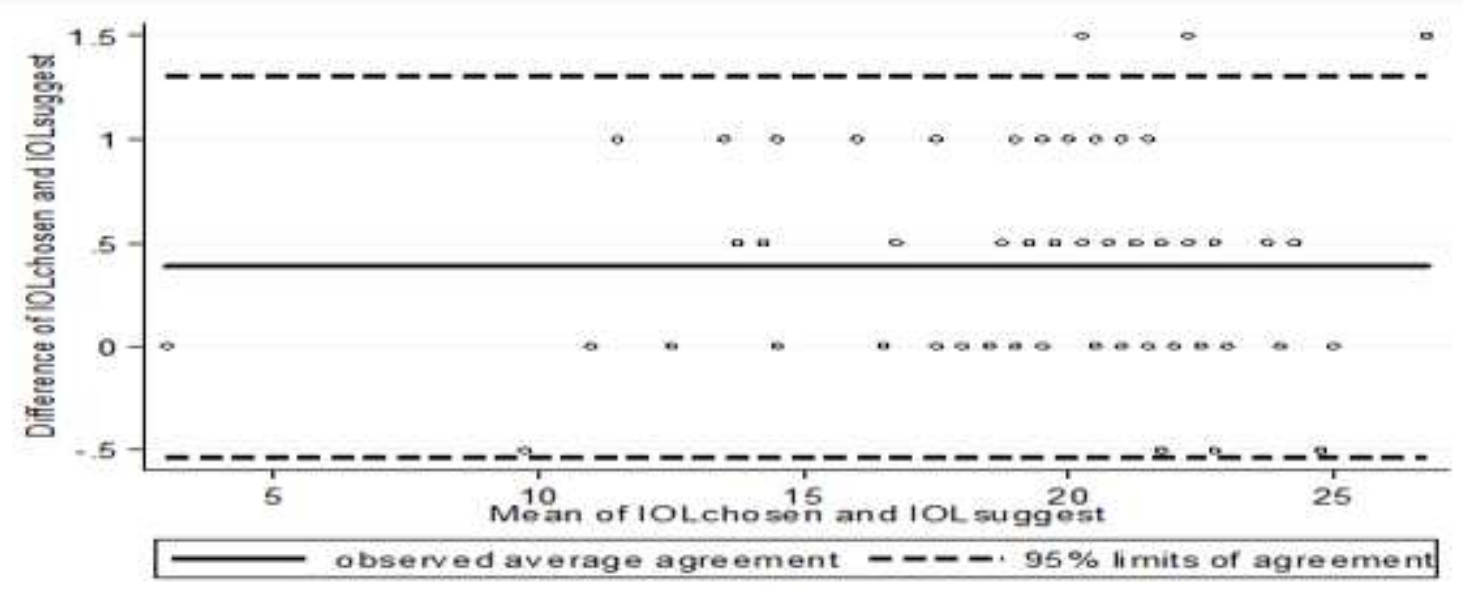

\section{Figure 1}

Bland-Altman analysis shows the difference between the IOL power derived from both devices is plotted against the mean for the two devices. The dotted lines represent the $95 \%$ limits of agreement. 